\title{
MEASUREMENT AND PREDICTION OF CENTRIFUGAL COMPRESSOR AXIAL FORCES DURING SURGE - PART 2: DYNAMIC SURGE MODEL
}

\author{
Enrico Munari \\ Università degli Studi di Ferrara \\ Ferrara, Italy \\ Klaus Brun \\ Southwest Research Institutes \\ San Antonio, TX, USA
}

\author{
Mirko Morini \\ Università degli Studi di Parma \\ Parma, Italy \\ Sarah Simons \\ Southwest Research Institutes \\ San Antonio, TX, USA
}

\author{
Michele Pinelli \\ Università degli Studi di Ferrara \\ Ferrara, Italy \\ Rainer Kurz \\ Solar Turbines Incorporated \\ San Diego, CA, USA
}

\section{ABSTRACT}

The force acting on centrifugal compressors is an important parameter to be considered throughout the operating life of these turbomachines. When the compressor is operating in surge conditions these forces can become highly dangerous for the mechanical and aerodynamic structures. This instability is usually avoided in industrial applications but the anti-surge system may not react in time when emergency shutdowns or power failures take place. During these rapid transients, surge can develop, generating unsteady forces which can harm the close clearance components of the compressor. Therefore, the capability to predict the characteristics and the dynamics of these surge forces would allow the estimation of the off-design fatigue cycles produced on these components by surge. Currently, no validated method exists to predict the frequency and amplitude of the surge forces and determine the potential damage of these components.

In this paper a lumped parameter model, developed by using the bond graph approach to predict the dynamic surge fluid-dynamic oscillations, is presented. The model requires the geometry and the steady-state performance maps of the compressor as inputs, together with the piping system configuration characteristics.

The simulator, is provided with a supplementary tool to estimate the axial force frequency and amplitude, taking into consideration all the contributions to the axial fluid-dynamic thrust, the stiffness-damping of the thrust bearing and the mass of the rotor.

The model was tuned and validated using the test case axial force data from the Southwest Research Institute facility. The model has shown good agreement with the experimental results which implies that it can offer significant information about the severity of a surge event and the quantification of the machine performance losses together with possible damage to the close clearance components. This study is a first important step that can lead to schedule optimization for maintenance and repair activities.

$\begin{array}{ll}\text { NOMENCLATURE } \\ A & \text { area } \\ C & \text { compliance } \\ C_{\mathrm{fc}} & \text { core rotation factor } \\ C_{\mathrm{p}} & \text { specific heat } \\ c & \text { damping coefficient } \\ D & \text { diameter } \\ F & \text { force } \\ f & \text { skin friction coefficient } \\ G & \text { half of the mass flow rate at the } \\ & \text { compressor curve peak } \\ g H & \text { impeller work } \\ H & \text { half of the difference between pressure } \\ & \text { ratio at peak and at zero mass flow } \\ & \text { rate } \\ I & \text { inertance } \\ K & \text { valve coefficient } \\ k & \text { stiffness } \\ L & \text { length } \\ M & \text { mass } \\ \dot{m} & \text { mass flow rate } \\ N & \text { rotational speed } \\ p & \text { pressure } \\ p r & \text { pressure ratio } \\ R & \text { resistance } \\ R & \text { gas constant } \\ r & \text { radius } \\ S & \text { source }\end{array}$




$\begin{array}{ll}S & \text { bearing clearance } \\ T & \text { temperature } \\ t & \text { time } \\ V & \text { volume } \\ v & \text { specific volume } \\ W & \text { generic thermodynamic work } \\ w & \text { velocity } \\ x & \text { spring deformation } \\ \alpha & \text { expansion coefficient } \\ \gamma & \text { specific heats ratio } \\ \Delta p & \text { pressure difference } \\ \eta & \text { efficiency } \\ \rho & \text { density } \\ \omega & \text { angular velocity }\end{array}$

$\begin{array}{ll}\text { Subscripts } & \\ \text { amb } & \text { ambient } \\ \text { ax } & \text { axial } \\ \text { B } & \text { bearing } \\ \text { C } & \text { compressor } \\ \text { corr } & \text { corrected } \\ \text { D } & \text { duct } \\ \text { damp } & \text { damping } \\ \text { disch } & \text { discharge } \\ \text { e } & \text { effort } \\ \text { el } & \text { elastic } \\ \text { exp } & \text { expansion } \\ \text { f } & \text { flow } \\ \text { fluid } & \text { fluid-dynamic } \\ \text { h } & \text { hub cavity } \\ \text { I } & \text { inertance } \\ \text { id } & \text { ideal } \\ \text { in } & \text { inlet section } \\ \text { is } & \text { isentropic } \\ \text { max } & \text { maximum } \\ \text { mom } & \text { momentum } \\ \text { out } & \text { outlet section } \\ \text { P } & \text { plenum } \\ \text { p } & \text { polytropic } \\ \text { pis } & \text { balance piston } \\ \text { rot } & \text { rotor } \\ \text { sh } & \text { shroud cavity } \\ \text { st } & \text { static } \\ \text { suct } & \text { suction } \\ \text { V } & \text { valve } \\ & \end{array}$

\section{INTRODUCTION}

Compressors are frequently used, especially in oil and gas applications, to complete processes [1-3]. Among the many types of compressors, a significant role is certainly played by centrifugal compressors that are often used in the process pipeline industry, offshore industry and chemical process industries as well. This is mainly due to their compact size, efficiency, and large tolerance to process fluctuations (from the large lower pressure processes to the high-pressure ones), high reliability and generally limited maintenance costs [4].
When analyzing the functionality of a centrifugal compressor, it is not sufficient to focus on aerodynamic performance as the trustworthiness of the mechanical system components also needs to be evaluated. In fact, the close clearance components of a centrifugal compressor are essential for the integrity of the system, so their wear grade and predicted operational life have to be monitored. It is clear that the components which define the fluid-dynamic path of the compressor can also influence these parameters. Interactions between the fluid, rotor and stator can vary significantly with the compressor's working conditions, since they mainly depend on the operating pressure ratio and mass flow - which define the operating point position on the compressor map. These interactions are the main factors responsible for the generation of forces which can damage some compressor components [5].

The prediction of these forces is indisputably convenient in order to schedule repairs or maintenance, thus allowing significant savings in terms of costs and preventing unpredicted production losses.

The bearing system, which constrains the rotor movement, is subject to the generated aerodynamic and mechanical force. It follows that the thrust bearing is one of the most critical elements, since its design and possible damage are evaluated by considering the thrust acting on it [6]. The evaluation of these loads plays a fundamental role, especially for centrifugal compressors, and can provide an accurate prediction of the bearing life.

As mentioned above, centrifugal compressors are frequently chosen in oil and gas applications. These industrial processes usually require compressors with low pressure ratios per stage, since the operating range needs to be as large as possible (the compressor curve is generally flatter), thus from very high flows (stonewall) to low flows (near surge) [7]. Choke conditions, in some circumstances, cause drop in efficiency but do not usually represent a risk for the reliability of the mechanical components of the unit. Surge is instead recognized as a drastic event which negatively affects the performance of a compressor [8] generating cyclic mass flow and pressure pulsations and thus impeding the turbomachinery from carrying out its work and guaranteeing the designed thermodynamic parameters of the process. Although surge is now effectively prevented in industry thanks to special dedicated control systems (surge avoidance systems controlling recycle circuits - in the case of compressed gases - or blow off valves - in the case of compressed air), some unpredictable transitory events or power failures can generate this phenomenon before the surge avoidance systems can react. When surge occurs, the aerodynamic features of the compressor completely change as does the axial thrust which can then severely damage the structures belonging to the compressor, and subsequently cause its destruction.

It is clear that, to correctly predict the axial thrust discharged on the thrust bearing, especially during surge, it is necessary to estimate the thermodynamic performance of the compressor during this instability. 
This paper presents a dynamic model for predicting the performance of a compressor installed in a piping system. This work represents the further development of the basic model presented in the preceding work [9] and is carried out in partnership with Southwest Research Institute (SwRI) and Solar Turbines Inc. The model is able to estimate the frequency and the amplitude of the mass flow rate and pressure oscillations, upstream and downstream of the compressor, during surge. The model was developed in Matlab/Simulink ${ }^{\circledR}$ environment and the main characteristic is its modularity. The model also has a specific additional tool, dedicated to the calculation of the axial aerodynamic force and the resultant thrust acting on the bearing.

The approach used for the dynamic modeling is that of bond graphs, which is not a novel methodology, but has been used by very few authors for investigating compressor surge (and its effect). Clearly, this increases the originality of this paper, which is also largely due to its main topic, i.e. the axial force during surge. In a previous work by the authors [9], an early preliminary (very simplified) version of the model has been shown to clearly predict deep surge thermodynamics, after proper tuning, in an axial-centrifugal compressor. In this paper, the advanced version of the model is also shown to provide high agreement with two-stage centrifugal compressor thermodynamic test data.

Moreover, the validation of the axial force prediction is addressed for the SwRI compressor tested.

In the following sections of the paper a brief literature review of the main works related to these topics, a description of the model, also showing the main results utilized for its validation (based on the experimental results obtained at the SwRI test facility), and a sensitivity analysis are presented.

\section{LITERATURE REVIEW}

In this section, a brief literature review on the modeling and control of surge and the axial force calculation (in both the experimental and modeling field) is reported.

\section{Surge model}

The importance of modeling the dynamic behavior of compressors or, more generally, of compression systems is due to the model's ability to predict, during operation, the occurrence of off-design conditions, instabilities and thus dangerous events. The reliability of these predictions assumes a fundamental role in (i) the design of the compressor (or system components) and (ii) the improvement of the control systems, in particular those associated with compressor instabilities, such as stall and surge.

Whereas rotating stall has two- or three-dimensional features, surge can be interpreted as a one-dimensional phenomenon, and for this reason can be simulated with the use of 1D tools. Many authors have developed dynamic models for predicting performance during compressor instability. All of these models have different features but many of them are based on a common root, which is the Greitzer model [11]. An important example of these surge models is that developed by
Morini et al. [12], not only for the model concept description, but also for its exhaustive literature review, approximately up to the early 2000 s.

Subsequently, many other authors have implemented new models. An example is the work of Galindo et al. [13] who carried out a campaign on a centrifugal compressor for automotive engines by modeling its behavior during surge and trying to predict how the compressor downstream volume affects the results. They developed a reliable compressor model (according to the Greitzer model) which was implemented in a pre-existing 1D gas-dynamic model of the piping system.

In the work of Vepa [14], an unsteady nonlinear model was presented. That model was an extension of the Moore and Greitzer model [15] and aimed to control surge and rotating stall by actively stabilizing the compressor. The results showed that the controller was successfully able to inhibit surge and stall.

A robust modeling technique, which is still used (as also reported by Day [16]) is the parallel compressor approach. This theory was proposed for the first time by Pearson and McKenzie [17] and allows the study of the inlet distortions by treating the compression system as two compressors, operating in parallel, which discharge the same static pressure [18]. This approach has been used in the past by many authors, for example in Mazzawy's work [19]. Nowadays, it is still a powerful solution, since it is a potential additional implementation to some classic surge models, to take into account also the effect of the inlet distortions [20].

It is clear that in any dynamic model, a significant role is played by implemented compressor characteristic curves. A comparison between different ways of modeling the compressor characteristics was presented by Grong [21]. In that work, dedicated to the control of surge, the close coupled valve and the drive torque actuation control methods were compared. Their potential advantages/limitations were highlighted, identifying the possible measurement time delay as a negative factor which made the controllers unable to react in a suitable amount of time to rapid transients. Another study, focused on the modeling of compressor performance during surge, is that presented by Belardini, et al. [22], in which the authors highlighted the importance of knowing the compressor characteristic curves in reverse flow. They developed and compared a 1D model and a Computational Fluid Dynamic (CFD) based method to predict the second quadrant compressor curves (i.e. the compressor performance in reverse flow conditions), emphasizing the fast operation of the former (more suitable for the standard production sector) compared to CFD, which was instead shown to be more powerful, allowing deeper insight into the fluid structures (in particular, blockage and secondary flows) at very low flow rates - the range in which the 1D model did not show good agreement with experimental data.

In the work of Leufvén and Eriksson [23], a compressor flow model was developed for simulating the behavior in choke, normal and surge operating conditions of an automotive compressor. This model was validated against a large 
automotive compressor map. The authors used the ellipse model structure to extrapolate data to small turbo speeds, which are not usually provided on the manufacturer datasheet.

Recently, two interesting works on surge modeling [24] and active surge control [25] were carried out. In the work of Kilchyk, et al.[24] a lumped parameter model was applied to a radial compressor, including the effects of heat transfer, whereas Yoon et al [25] developed and validated a control algorithm based on the actuation on the impeller tip clearance using magnetic bearings.

Based on this review, it is clear that that the modeling of surge has achieved a certain degree of robustness, even if the experimental results cannot always be exactly replicated. The progress in this sector can be represented by the improvement of the detection, avoidance and control systems.

\section{Axial force}

Whereas literature offers accurate methods for evaluating radial thrust, it is less precise and complete regarding the estimation of the axial thrust, due to the high number of factors which influence this prediction. This implies that the evaluation of axial thrust still cannot count on a universal and completely reliable method. This section of the paper lists the experimental, modeling and even purely theoretical works dealing with axial thrust evaluation.

The study of axial thrust on turbomachinery began approximately around the 1960s, and one of the first and most representative works, although focusing on centrifugal pumps, was presented by Domm and Zilling [26]. Later, since the works of Kurokawa and Toyokura [27] the effect of radial inward leakage flow on axial thrust in turbomachines also began to be analyzed. They theoretically and experimentally examined the flow between the impeller and the casing of a radial turbomachine finding the influence of some parameters such as angular momentum and axial spacing between the rotor and the casing.

After the study of Mazzawy [28] who introduced the problem of structural loads in gas turbines during surge, the evaluation of the forces generated in turbomachines acquired more interest. In fact, he was one of the first to offer a simple model for estimating the impact of surge force on engine structures. Bondarenko, et al. [29] developed a set of equations considering the flow between the casing and the impeller; the results showed good agreement with the experimental data.

Lately, Baldassarre, et al. [30] have developed 1D software to estimate radial pressure distribution along impeller surfaces, and subsequently, the total axial thrust acting on the rotor; they validate the model using the experimental data from two high pressure centrifugal compressors tested at full load. In that work they used the correlation of Da Soghe, et al. [31].

More recently, Moore, et al. [5] have tried to approach this topic by means of computational fluid dynamics. They applied for the first time CFD methods to study the rotordynamic forces on a centrifugal compressor. They used a particular meshing technique which allowed (i) a significant time saving and (ii) adequate correlation to the recorded data. This study revealed that most of the destabilizing force occurred in the shroud gap.

In the work of Han and Cizmas [32] a numerical method, not validated with experimental data, for the calculation of the axial thrust of a centrifugal compressor was presented. In that investigation both the flow through the impeller and the leakage flow on the back side of the wheel were simulated for a single operating point.

Relevant work has been carried out, in recent years, by Bidaut and Dessibourg [33]. They reported remarkable thrust measurements on different types of compressors. They also described the main concepts and key points that need to be considered for the correct evaluation of axial thrust. They conducted the study focusing on the parameters which affect this calculation the most. They also reported a set of equations, based on literature, for achieving an accurate estimation of the axial loads. Based on their history review, the experimental evaluation of thrust is quite a recent activity. Some measurements were carried out in the 1990s [34] when a study on rotordynamic stability was performed, focusing on the influence of thrust brakes. Later, Bidaut and Baumann performed other measurements [35] in a 655 bar compressor, recording data by means of a thrust collar in the gear and also taking the rotor damping.

For high pressure applications, the thrust calculation should carefully take into consideration the phenomena occurring along the side cavities, as shown in the work of Petry, et al. [36] and Roy, et al. [37]. Many authors have carried out studies, even without the specific aim of calculating axial thrust, to analyze the flow in the side chambers [38, 39]. These examples may be very useful for developing a mathematical algorithm to evaluate in detail the pressure distribution along the gaps for a high pressure compressor. One of the main references with extensive dissertation of rotating flows in the cavities is the book published by Childs [40].

Finally, a last important study to mention is that of Lüddecke, et al. [6], in which the rotor motion of a turbocharger was analyzed. In that paper the thrust bearing reaction forces were also measured under various engine operating conditions and a model was proposed. The results were compared with the recorded data, and the approach used has proved to be successful for a single-stage waste gate turbocharger.

This section highlights that, currently, the methodology used to calculate centrifugal compressor axial thrust, especially for high-pressure applications, is still a field of research which needs to be explored, advanced and expanded. For this reason, this work aims to provide a significant contribution by offering the description and results of a brand new dynamic model with an additional tool for calculating the net axial thrust during surge.

\section{DYNAMIC MODEL}

The dynamic lumped parameter model was developed in Matlab/Simulink ${ }^{\circledR}$ environment and it was intended to be as modular as possible. This structure was achieved by means of the bond graph approach, which is a modeling technique that 
can be adopted in many engineering systems; an example is given in the work of $\mathrm{Hu}$ and $\mathrm{Li}$ [41]. The theory and basic concepts of the bond graph technique can be found in the work published by Brown [42].

Regarding the turbomachinery and energy system field, only a few authors [43-45] used this method, therefore it can be considered an original methodology for modeling the dynamic behavior of the compression piping systems during surge. This modeling technique is a powerful way of developing dynamic models, as demonstrated in [9], by comparing the model response with the experimental data from [46]. Once the correct graphic bond graph representation is complete, the causality assignment (i.e. a series of causes and effects which basically define the computation order of the efforts and flows) is simply regulated by the stroke of the bonds and the nature of the inputs. Therefore, the bond graph offers an intuitive way to obtain the differential equations governing the system.

\section{Compression system model}

The aim of the model is to simulate the dynamic behavior of a centrifugal compressor, installed in a given piping system, during its operating conditions, especially in surge.

The model is based on the hypothesis of ideal gas, however, since the future target of this advanced version of the model is the oil \& gas applications field, the simulator code was intended to be able to handle different types of gases and mixtures. For this reason, this improved version allows the assessment of the specific heat coefficients, the gas constants and the specific heat ratios [47], thus it is currently suitable for low-to-medium pressure applications. The constant coefficients which are useful for calculating the specific heat as a function of temperature, were found. By using the molecular weight of the gas as input, $R_{\text {gas }}$, and $\gamma$ were determined. This procedure was applied to several gases used in industry. Moreover, if a mixture is used in the model and the correct percentage fraction of gases is set, the characteristic gas constant of the mixture can be calculated.

To make the model suitable for industry (this project was carried out within the framework of the Gas Machinery Research Council activity), all these inputs, together with the geometrical inputs of the systems and steady-state curves of the compressor (or single-stages, see subsection "The compressor") are automatically uploaded in the Matlab workspace. This is done by means of a specific script which communicates with an Excel $\AA$ file. For the same reason, despite the complexity of the model, the computational cost was carefully taken into account. The typical elements to be implemented to simulate a real application are the valves, the ducts, the plenums and obviously the compressors. The independent construction of single modules (see Fig. 1), provides great modularity; which is the main characteristic required by an adequate model.

By using a simple layout for each element and by exploiting the bond graph theory, the main elements can be mathematically defined. Their graphic representation and bond graphs are illustrated in Fig.1.

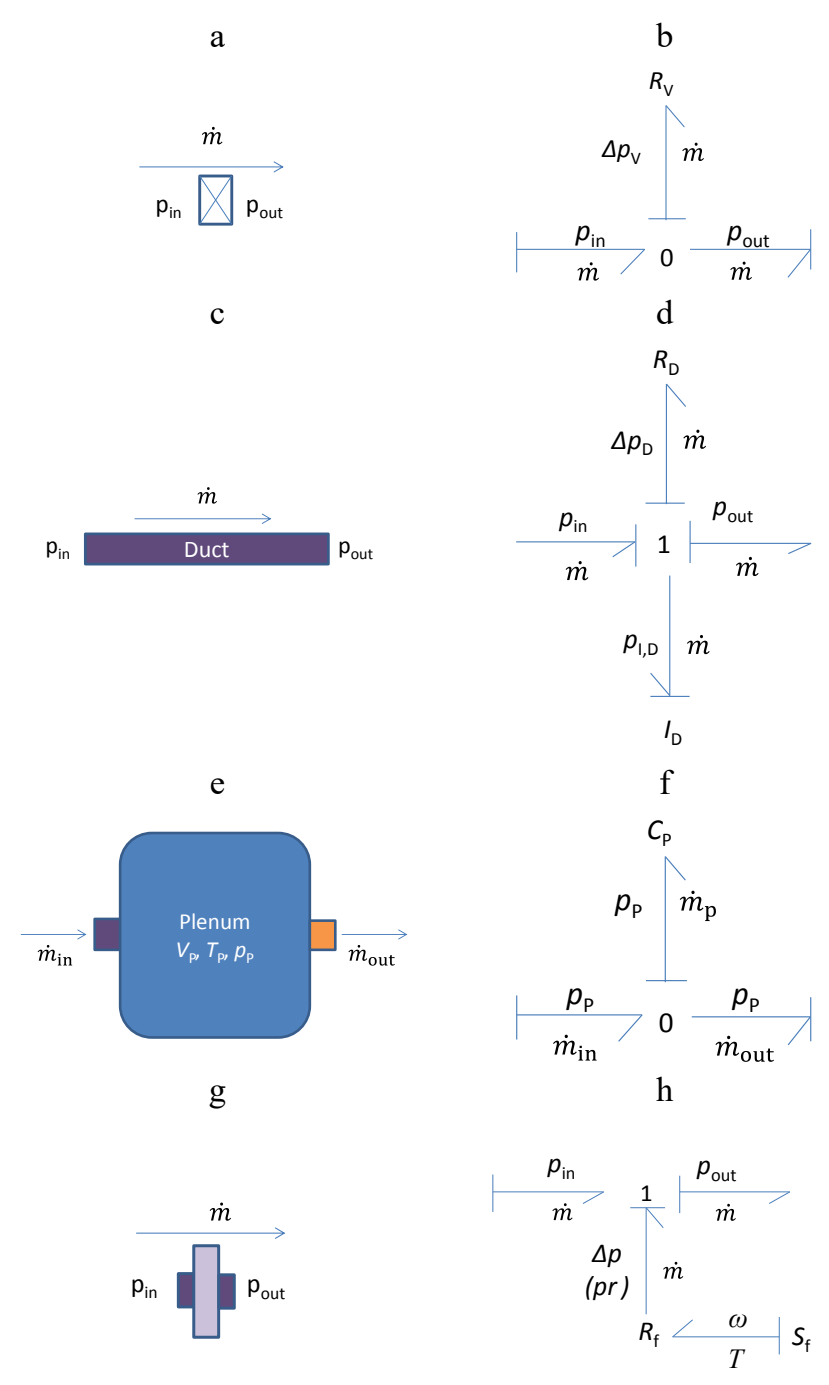

Fig. 1. Layout and bond graphs of the basic elements of the model

By using a simple layout for each element and by exploiting the bond graph theory, the main elements can be mathematically defined. Their graphic representation and bond graphs are illustrated in Fig.1.

\section{The inlet valve}

The valve, see Fig. 1a, is a dissipative element whose characteristics are embodied in the resistance $R_{\mathrm{V}}$, which is basically defined by the valve coefficient $K_{\mathrm{V}}$. This element is modeled in order to determine the pressure drop $\Delta p_{\mathrm{V}}$, given the mass flow rate, see (1a). In addition, a second type of valve (not shown) was modeled, in order to increase the modularity of the model, as an element which uses the same physical principle and equation but determines the mass flow rate, given the pressure drop, as shown in (1b). 
$\Delta p_{\mathrm{V}}=\frac{\dot{m}^{2}}{K_{\mathrm{V}}^{2} \bar{\rho}}$

$\dot{m}=K_{\mathrm{V}} \times\left( \pm \sqrt{\left|\bar{\rho} \Delta p_{\mathrm{V}}\right|}\right.$

In order to evaluate the valve coefficient $K_{\mathrm{V}}$, the modeling of this component behavior, in particular the quantification of the valve resistance $R_{\mathrm{V}}$, requires the use of algebraic correlations or, alternatively, constitutive relations of the valve (i.e. the characteristic curve of the valve, which can be implemented and read by the model). The bond graph of this element consists of a "1-junction" (Fig. 1b), linked to three bonds: one bond represents the resistance effect, and the other two represent the inlet and the outlet energy flux. In this element the outlet temperature is assumed equal to the temperature at the inlet (and vice versa in case of reverse flow) therefore, the increase of fluid entropy and also the heat exchange to the atmosphere are neglected

\section{The duct}

The duct, sketched in Fig. 1c, is an inertance energy storage element which also has a dissipative effect. Therefore, the characteristic parameters of this element are the resistance $R_{\mathrm{D}}$ and the inertance $I_{\mathrm{D}}$, which are used to calculate the mass flow rate as a function of the pressure at the inlet and at the outlet of the element, as shown in (2)

$\left\{\begin{array}{c}p_{\mathrm{I}}=p_{\text {in }}-\Delta p_{\mathrm{D}}-p_{\text {out }} \\ \frac{\mathrm{d} \dot{m}}{\mathrm{~d} t}=\left(\frac{p_{\mathrm{I}}}{I}\right)\end{array}\right.$

From this system we can obtain

$\frac{\mathrm{d} \dot{m}}{\mathrm{~d} t}=\frac{A}{L}\left(p_{\text {in }}-\Delta p_{\mathrm{D}}-p_{\text {out }}\right)$

where the pressure drop, $\Delta p_{\mathrm{D}}$, is calculated following equation (4):

$\Delta p_{\mathrm{D}}=f \frac{L}{D} \rho \frac{V^{2}}{2}$

The bond graph of the duct, see Fig $1 \mathrm{~d}$, is represented as a "1-junction" connected to the resistance $R_{\mathrm{D}}$, the inertance $I_{\mathrm{D}}$, the inlet and the outlet energy flux. Also in this case the outlet flow is assumed to have the same temperature as the inlet flow. The dynamic model for the pipe element is based on the lumped parameter approach, hence perturbation propagation time from one end of the pipe is not accounted for. This is a major assumption, particularly in dealing with compressor surge where wave propagation time is crucial. The present approach neglects this effect.

\section{The plenum}

The plenum, shown in Fig. 1e, was considered as an accumulator, i.e. a capacitive element with a characteristic compliance $C_{\mathrm{P}}$. This parameter is used to calculate the plenum pressure as a function of the mass flow rate at the inlet and at the outlet, as shown in (5). The dissipative effect generated at the inlet and the outlet can be considered negligible since the main effect of this component is to store energy.

$\left\{\begin{array}{c}\dot{m}_{\mathrm{P}}=\dot{m}_{\text {in }}-\dot{m}_{\text {out }} \\ p_{\mathrm{P}}=\frac{1}{C_{\mathrm{P}}} \int \dot{m}_{\mathrm{P}} \mathrm{d} t\end{array}\right.$

The derivative equation of the plenum pressure can thus be written as:

$\frac{\mathrm{d} p_{\mathrm{P}}}{\mathrm{d} t}=\frac{\gamma R_{\mathrm{g}} T_{\mathrm{P}}}{V_{\mathrm{P}}}\left(\dot{m}_{\text {in }}-\dot{m}_{\text {out }}\right)$

The temperature was also taken into account in this module. In fact, from the conservation of energy, the following equation was implemented in the model:

$\frac{\mathrm{d} T_{\mathrm{P}}}{\mathrm{d} t}=\frac{1}{m_{\mathrm{P}} C_{\mathrm{p}}}\left[\sum_{\text {in,out, } \mathrm{P}} \dot{m}_{\text {in,out }} C_{\mathrm{p} \text { in,out }} T_{\text {in,out }}+T_{\mathrm{P}} \alpha_{\mathrm{P}} V_{\mathrm{P}} \frac{\mathrm{d} p_{\mathrm{P}}}{\mathrm{d} t}\right]$

The bond graph of the plenum (Fig. 1f) consists of a " 0 junction" connected to a capacitance and to the inlet and outlet energy flux. The plenum, like the other elements of the model, is assumed to be adiabatic - this assumption is justified by the fact that the dynamics of fluid heat exchange are much faster than the piping system mass heat exchange.

\section{The compressor}

This element is treated as a resistance field which acts as a non-dimensional actuator disk (Fig. 1h). The general compressor representation used in this work is illustrated in Fig. 1g. The compressor calculates the pressure at the inlet, given the outlet pressure, the mass flow rate and the rotational speed. Moreover, it determines the temperature at the outlet (or at the inlet when backflow occurs) by using the isentropic efficiency curves (or the first law of thermodynamics).

Two types of compressor modules were implemented, so one or the other can be used depending on the performance data provided. If only the overall compressor maps of a multistage compressor are known, the compressor is modeled by the "Overall Compressor" module, otherwise, if the maps of each specific stage are known, the compressor can be modeled using the "Single Stage" module (by placing in series as many modules as the number of stages). Both of these modules read the performance curves of the compressor, i.e. the static to static pressure ratio and the isentropic efficiency, in terms of corrected rotational speed coefficient, $N_{\text {corr }}$, and corrected mass flow rate coefficient, $\dot{m}_{\text {corr }}$, expressed as 
$N_{\text {corr }}=\frac{N}{\sqrt{\gamma R T_{\text {in }}}}$

$\dot{m}_{\text {corr }}=\frac{\dot{m} \sqrt{R T_{\text {in }}}}{p_{\text {in }} \sqrt{\gamma}}$

This choice allows the assessment of the variation in compressor performance with the type of gas and changes to ambient conditions, except for humidity [48] [49] [3]. The performance curves implemented in the module derive from the experimental performance tests. A parametric procedure was applied considering the characteristic curves as quadratic, so that the three coefficients can be calculated. The ideal maximum mass flow rate value (at zero pressure ratio), and the value at the peak of the curve, i.e. at $p r_{\max }$, together with other significant parameters are expressed as a function of the rotational speed by using quadratic or third degree polynomial curves. These correlations are implemented in the model so that at each rotational speed, the three coefficients of the quadratic characteristic curve of the compressor can be obtained.

The same method was carried out with the isentropic efficiency curves. Therefore, the model uses the corrected curves obtained through the parametric study until the compressor is operating in the stable part of the curves. Then, when the operating point, calculated by the model, goes to the left of the characteristic curve peak, the model can adopt two different curves, as shown in Fig. 2: (a) the Moore and Greitzer cubic law, as also implemented in [50], or (b) the sudden pressure drop/rise. The first or the second alternative may be chosen depending on the type of effect the surge is expected to have generated, and depending on the type of characteristic curve the compressor is expected to have beyond the peak. The cubic law, which is also valid for reverse flow, allows the calculation of the pressure ratio as:

$p r_{\dot{\mathrm{m}}<0}=p r_{\dot{\mathrm{m}}=0}+H\left[1+\frac{3}{2}\left(\frac{\dot{m}}{G}-1\right)-\frac{1}{2}\left(\frac{\dot{m}}{G}-1\right)^{3}\right]$

where the main parameter to be calculated is the pressure ratio at zero mass flow rate:

$p r_{\dot{\mathrm{m}}=0}=\left(1+\frac{\eta_{\mathrm{is}_{\mathrm{m}}=0} g H_{\mathrm{id}_{\mathrm{m}}=0}}{C_{\mathrm{p}} T_{\mathrm{in}_{\mathrm{m}}=0}}\right)^{\frac{\gamma}{\gamma-1}}$

$=\left(1+\frac{\eta_{\mathrm{is}_{\mathrm{m}=0}} \pi^{2}\left(\frac{N}{60}\right)^{2}\left(D_{2}{ }^{2}-D_{1}{ }^{2}\right)}{C_{\mathrm{p}} T_{\mathrm{in}_{\mathrm{m}}=0}}\right)^{\frac{\gamma}{\gamma-1}}$

The temperature of the flow leaving the compressor is also calculated. In direct flow (and when the mass flow is zero) the temperature is calculated as
$T_{\text {out }}=T_{\text {in }}(p r)^{\frac{\gamma-1}{\gamma \eta_{\mathrm{p}}}}$

where the polytropic efficiency, $\eta_{\mathrm{p}}$, can be expressed as:

$\eta_{\mathrm{p}}=\frac{\ln \left(\frac{p_{\text {out }}}{p_{\text {in }}}\right)^{\frac{\gamma-1}{\gamma}}}{\ln \left(\frac{\left(p_{\text {out }} / p_{\text {in }}\right)^{\gamma / 1}-1}{\eta_{\text {is }}}+1\right)}$

In direct flow conditions, the isentropic efficiency is derived from the maps experimentally obtained, whereas at zero mass flow it is calculated following a procedure that considers the losses due to incidence and friction, as reported in [51] and also utilized by Jiang, et al. [52], that considers the losses due to incidence and friction.

When the compressor goes into surge the concept of efficiency can be misleading so in this work a different strategy was used to evaluate the temperature. In fact, the first law of thermodynamics was applied to the compressor, also considering the expansion effect that the gas experiences in reverse flow, along the compressor. The expansion work is calculated as:

$W_{\text {exp }, \mathrm{C}}=p_{\text {out }} v_{\text {out }}-p_{\text {in }} v_{\text {in }}$
$\cong \frac{p_{\text {out }}-p_{\text {in }}}{\rho_{\text {out }}}=\frac{p_{\text {out }}}{\rho_{\text {out }}}\left(1-\frac{p_{\text {in }}}{p_{\text {out }}}\right)=R_{\mathrm{g}} T_{\text {out }}\left(1-\frac{1}{p r_{\text {i }<0}}\right)$

The temperature of the flow leaving the compressor is then:

$T_{\text {in }}=T_{\text {out }}+\frac{g H_{\mathrm{id}_{\mathrm{m}<0}}-W_{\text {exp }, \mathrm{C}}}{C_{\mathrm{p}}}$

Where $T_{\text {out }}$ is the temperature entering the compressor. The impeller work, $g H_{\text {id }}$ is calculated using the Euler equation. Therefore, this module can simulate both vaneless and vaned diffuser compressors.
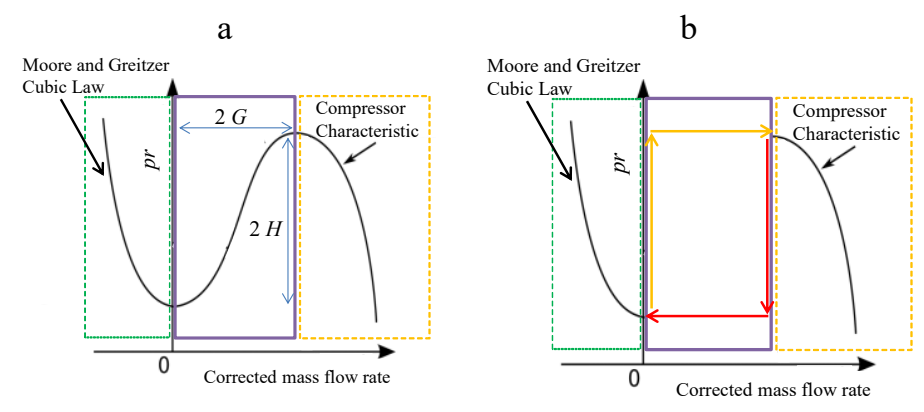

Fig. 2. Compressor curves in surge conditions 
The difference between these two cases (vaneless and vaned diffusers) is the calculation method of the impeller work in reverse flow since the impeller entering flow incidence is substantially different. In a vaneless diffuser [53] the flow is assumed to enter with a perfect radial direction at the impeller tip (this is a simplistic assumption since it is feasible that the volute and return channel might affect the flow pattern - more in-depth analysis will be carried out in future works) whereas in a vaned diffuser the incidence depends on the vane angles.

The concept and methodologies explained above are applied to both the "Overall Compressor" and the "Single Stage" module. However, in the overall compressor module only the overall maps of the compressor are given as inputs. In that module a procedure is then applied to estimate the interstage values of pressure and temperature, a problem which other authors have faced in different ways [57,58]. This additional calculation is not required when analyzing the compression system dynamics, but was implemented because the estimation of the pressure and temperature at the inlet and outlet of each impeller becomes fundamental when calculating the axial fluid-dynamic thrust.

\section{Axial thrust calculation}

The axial load in a compressor is generated by the accumulation of the thrust of the impeller (or impellers). When calculating the forces, a fundamental role is played by the geometrical dimension of the rotor diameters and the seal clearances in addition to the balance piston geometry and the operating conditions. As already mentioned above, the evaluation of the resultant force strongly depends on the pressure, mass flow rate and temperature at the inlet and at the outlet of each impeller. Therefore, before applying the calculation it is important to count on a reliable compression system model.

Figure 3 shows the typical layout of single "in-line" impeller and the pressure contributors. The axial thrust calculation is implemented in the model as a separate tool and is carried out at each impeller (if the subject is a multistage compressor), since the thermodynamic conditions at the compressor inlet and outlet are not sufficient to obtain a good estimation. The following considerations, are valid for enclosed impellers - semi-open impellers, not investigated in this paper, are prone to higher axial forces since they only have one disk, and thus the axial thrust balance is more critical (higher risk of damage). The resultant fluid-dynamic axial force, $F_{\text {ax,fluid, }}$ is calculated by considering the main contributions [54-56] (see Fig. 3), which are basically the inlet and outlet static pressure, the pressure along the disk cavities (i.e. at the shroud and at the hub), the axial momentum variation between the inlet and the outlet, and finally, the pressure distribution on the balance piston. Based on the layout of Fig. 3 the resultant equation for each perfectly radial impeller is:

$F_{\text {ax } \text { fluid }}=F_{\text {st,in }}+F_{\text {sh }}+F_{\text {mom }}-F_{\mathrm{h}}-F_{\text {pis }}$

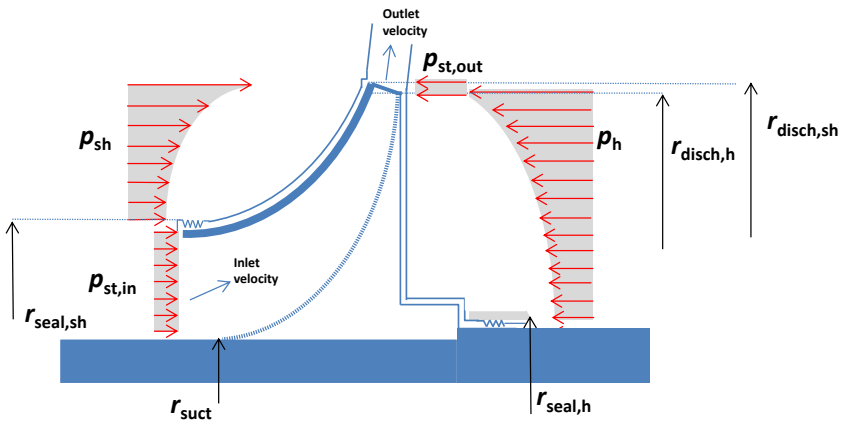

Fig. 3. Force contributions in a single impeller

The most difficult contributions to estimate are $F_{\mathrm{sh}}$ and $F_{\mathrm{h}}$, which derive from the flow fluid-dynamics in the hub and shroud cavities. These two forces are the result of a static force, constant along the disks, and a kinetic force which varies with the radius. By applying the radial equilibrium in a control volume of a cavity, it is possible to obtain the derivative equation of the pressure with respect to the radius:

$\frac{\mathrm{d} p}{\mathrm{~d} r}=\rho r C_{\mathrm{fc}}^{2} \omega^{2}$

By integrating this equation, the pressure value at a specific radius of the cavity (shroud or hub) is:

$p_{\text {sh }, \mathrm{h}}(r)=p_{\text {disch }}-\rho_{\text {disch }} \omega^{2} r_{\text {disch }}^{2} \frac{C_{\mathrm{fc}}^{2}}{2}\left[1-\left(\frac{\mathrm{r}}{r_{\mathrm{disch}}}\right)^{2}\right]$

Equation (18) is valid for both hub (subscript h) and shroud (subscript sh) cavities. This equation strongly depends on the core rotation factor, $C_{\mathrm{fc}}$, which is very hard to estimate without weighing down the model with additional empirical formulations. To the knowledge of the Authors, there are no robust empirical ways as yet to determine this parameter with a reasonable computational cost. Moreover, to be very accurately calculated at each desired radius, the values of many other parameters, such as friction coefficients, roughness, etc., need to be known, which is very hard in industrial practice.

Since this model is dedicated to applications with medium to low pressure values, and since the model was thought to provide a reliable indication in a reasonable simulation time, and with the requirement of knowing only the main geometric parameters, the core rotation factor is kept constant at each cavity. Based on literature, this method is very often used in industrial practice. Once the pressure distribution is calculated with (18) at $n$ discretization points along the gaps, the force contribution is given by:

$F_{\mathrm{sh}, \mathrm{h}}=\sum_{\mathrm{i}=1}^{\mathrm{n}} p\left(r_{\mathrm{i}}\right)_{\mathrm{sh}, \mathrm{h}} A(i)_{\mathrm{sh}, \mathrm{h}}$ 
Where $A(i)$ is the area of the annulus between two consecutive discretization points.

Another important contribution comes from the static force at the inlet which can be easily obtained as:

$F_{\text {st, in }}=\pi\left(\mathrm{r}_{\text {seal }}{ }^{2}{ }_{\text {sh }} r_{\text {suct }}{ }^{2}\right) p_{\text {suct }}$

where $p_{\text {suct }}$, which basically acts between the shroud seal radius and the impeller eye radius, is the static pressure at the impeller suction. A similar consideration can be made for the pressure at the impeller discharge.

The variation in axial momentum is also important to evaluate. This contribution can be expressed as:

$F_{\text {mom }}=w_{\text {ax, suct }} \dot{m}-w_{\text {ax }, \text { disch }} \dot{m}$

Finally, the force acting on the balance piston surface needs to be considered. A simple assumption adopted is the uniformity of the pressure distribution on its faces, which are exposed to the discharge pressure and the suction pressure of the impeller. The equation used is:

$F_{\text {pis }}=\frac{\pi}{4}\left(D_{\text {pis }}^{2}-D_{\text {seal, }}^{2}\right) p_{\text {suct }}$

All these formulae allow the determination of the fluiddynamic axial thrust which develops in a single impeller during operation. In case of multistage compressors, the same method needs to be applied to each stage and the overall thrust will be the sum of the axial force of each stage. In order to evaluate the severity of the surge forces, the characteristics of the bearing and the rotor also have to be taken into account.

\section{The thrust bearing and the mass of the rotor}

The thrust bearing is the component which provides axial positioning for the rotating components and it also withstands axial forces not offset by the balance piston during compressor operation - these forces arise from the change in magnitude of the rotor axial velocity. Therefore, its characteristics have to be contemplated when computing its constraint force in order to evaluate the potential damage caused by a surge event. This force strongly depends on the stiffness, and damping characteristics of the bearing. The first value should be provided by manufacturers whereas the second one is quite hard to estimate (as, in general, for many mechanical systems). Therefore, a suitable modeling layout of a thrust bearing is a mass-spring-damper system, on which an external force (i.e. the fluid-dynamic force) is applied, where the mass element is the rotor, i.e. the compressor shaft with the impellers. The system (see Fig. 4a) can be modeled by adopting the bond graphs, as shown in Fig. 4b. Therefore, the resultant force is given by:

$F_{\text {rot }}=F_{\text {ax }}-F_{\text {el }}-F_{\text {damp }}$

Where $F_{\text {el }}$ and $F_{\text {damp }}$ are, respectively, the elastic force and the damping force of the bearing whereas $F_{\text {rot }}$ is the force generated by the rotor mass. The acceleration of the rotor can then be found as:

$\ddot{x}_{\text {rot }, \mathrm{ax}}=\frac{F_{\mathrm{ax}, \mathrm{fluid}}-F_{\mathrm{el}}-F_{\mathrm{damp}}}{M_{\mathrm{rot}}}=\frac{F_{\mathrm{ax}, \text { fluid }}-k x-c \dot{x}}{M_{\mathrm{rot}}}$

The above equation provides a fairly good approximation of the net axial thrust acting on the thrust bearing, which is its reaction force, calculated as:

$F_{\mathrm{ax}, \mathrm{B}}=\left|-F_{\mathrm{el}}-F_{\text {damp }}\right|$

\section{MODEL APPLICATION: SWRI TEST CASE}

The model was applied to the test bench installed at the Southwest Research Institute. Therefore, the model reliability was proven by means of a validation with the experimental data. The basic geometry dimensions of the SwRI piping system layout are shown in Tab.1. It consists of a complex system built to generate surge conditions and then to study the dynamic response of the compressor to rapid transients. The electric motor driven compressor processes the air at ambient conditions and the mass flow is regulated by means of the 6 inch control valve. Additionally, a 1-inch manual valve can be opened when needed to allow for finer opening/closing steps of the control valve, thereby providing better control of the compressor's approach to surge (the P\&ID of the system can be found in the paper part $1[10])$.

The compressor is a multistage centrifugal compressor equipped with two radial impellers and two vaneless diffusers. The overall compressor maps are presented in Fig. 5, in terms of pressure ratio versus actual volumetric flow, at different rotational speeds $(7000,9000,10,000,11,000,12,000$ and $13,000 \mathrm{rpm})$. The isentropic efficiency curves are not provided but significant values of efficiency are known: the peak is equal to $65 \%$ whereas, during surge, it ranges from $45 \%$ to $50 \%$.

For this specific application, the model is implemented using the layout illustrated Fig. 6a. The intake duct is preceded by an inlet valve, which is useful for providing stability to the model. The pressure drops caused by this element (which is not present in the experimental test loop) are set to infinitesimal by adjusting the valve coefficient.

The compressor downstream of the experimental test loop is evaluated as a plenum with a capacity equal to the volume of the ducts between the compressor and the control valve. The remaining part of the test loop (downstream of the compressor) is basically considered as pressure drops by tuning the outlet valve coefficient.

Fig. $6 \mathrm{~b}$ illustrates the bond graph of the entire piping system. The overall bond graph simply derives from the connection between the bond graphs of each element selected for the simulation. From this figure it is possible to understand the causality assignment adopted by the model more clearly in order to solve the differential equations and calculate the thermodynamic parameters. The boundary conditions of the system are the ambient pressure and temperature (the values at 
which the test was conducted). The simulator was set with an ODE 45 solver (which is based on the Runge-Kutta method for solving ordinary differential equations) with very small step size and tight tolerances in order to give maximum reliability to the results, although partially sacrificing the speed of the simulator.

\section{SIMULATIONS AND RESULTS}

The model allows the compressor performance to be simulated at any rotational speed. The validation of the results was carried out by comparing the thermodynamic and force data results, at 13,000 rpm, obtained in the experimental facility and resulting from the model. Some additional relevant plots are also shown in order to demonstrate the reliability and accuracy of the thermodynamic and axial force calculation of the model. In the model, the compressor achieves the desired speed, through a linear accelerating ramp from an initial value (usually set to $5000 \mathrm{rpm}$ ). The outlet valve starts to close after a certain amount of time (usually set to $20 \mathrm{~s}$ ) by linearly reducing the valve coefficient to a suitable value to generate mild surge. The results of a typical model simulation, in terms of compressor operating point path and discharge pressure of each impeller, are presented in Fig. 7a and Fig. 7b, respectively.

The characteristic curve resulting from simulation is expressed, in Fig. 7a, as the pressure ratio versus the mass flow rate. After the initial ramp, from $5000 \mathrm{rpm}$ to $13,000 \mathrm{rpm}$, the stall ramp obtained by closing the outlet valve can be identified. This curve clearly resembles the experimental one, verifying the reliability of the model and parametric procedure applied although the steady-state curve are used as inputs, the agreement with the model may not be obvious and strongly depends on the procedure; in this case the parametric procedure was successful at all rotational speeds. Finally, surge is obtained, with the characteristic mild oscillations of the operating point around the characteristic curve peak.

The simulation lasts $100 \mathrm{~s}$ and the trends of the discharge pressure of the two impellers, in Fig. 7b, show the typical increase due to the velocity increase in the first $15 \mathrm{~s}$, and due to the closure of the valve between the range 20-29 s.

After the speed increase, and closure of the valve, mild surge fluctuations occur during the remaining time of the simulation.

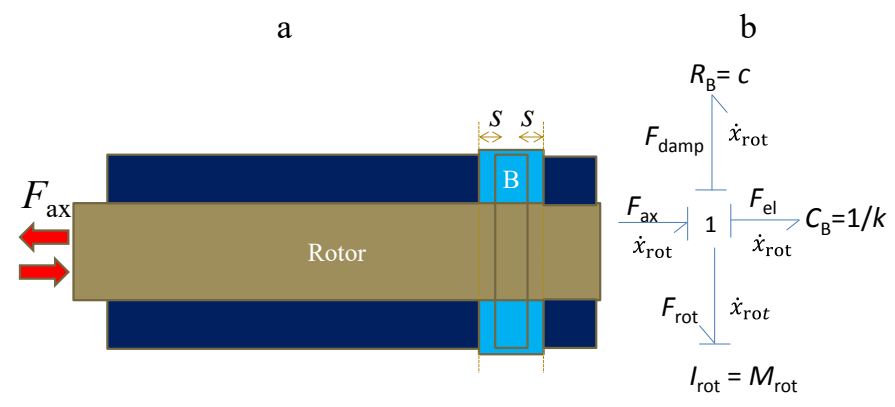

Fig. 4. Scheme and bond graph of the bearing system

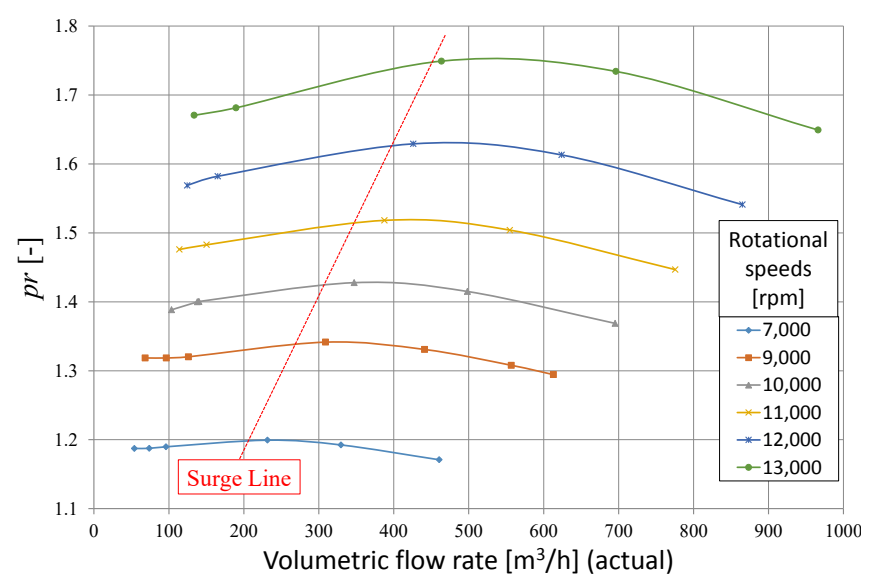

Fig. 5. Characteristic performance maps of the compressor tested

Tab. 1 Characteristic piping system dimensions

\begin{tabular}{|c|c|c|}
\hline & $\begin{array}{c}\text { Length } \\
{[\mathbf{m}]}\end{array}$ & $\begin{array}{c}\text { Volume } \\
{\left[\mathbf{m}^{\mathbf{3}}\right]}\end{array}$ \\
\hline Pipes upstream of the compressor & 3.00 & 0.07 \\
\hline Pipes downstream of the compressor & 14 & 0.21 \\
\hline
\end{tabular}

a

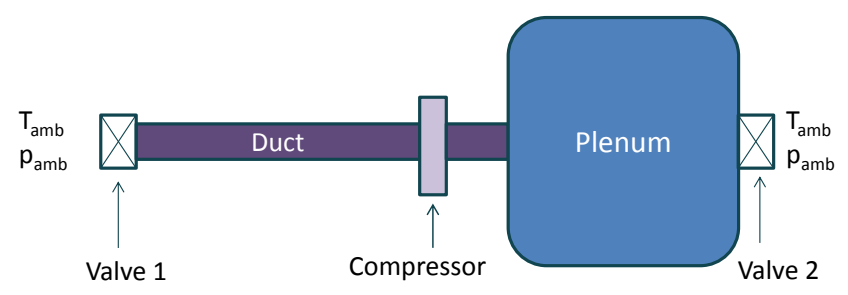

b

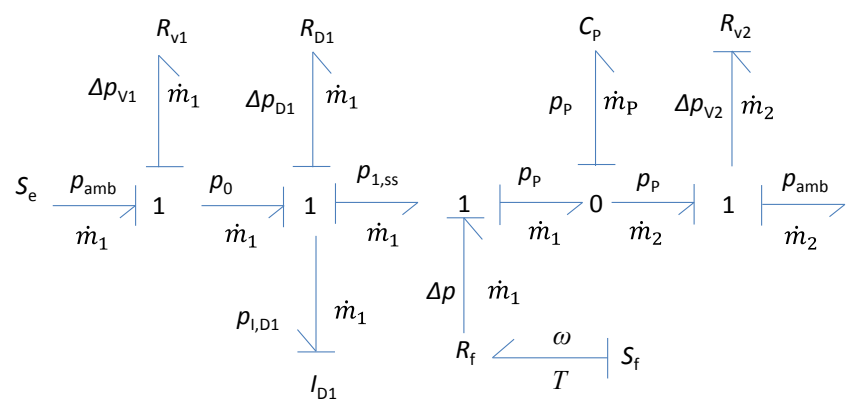

Fig. 6. Piping system: layout (a) and bond graph (b)

Simulations were also carried out at other compressor speeds. Table 2 reports a comparison between model and experimental results of the pressure ratio values at surge onset at the different rotational speeds tested. The model thermodynamic results are shown to be very close to the test case data at any rotational speed simulated. As can be seen, the percentage error is negligible (maximum error is $0.8 \%$.). 
The axial thrust acting in the thrust bearing is calculated by a separate module as a function of the mass of the rotor, the stiffness and the damping effect of the bearing system. The resultant axial force generated by the compressor at 13,000 rpm, and supported by the bearing, is shown in Fig. 8.

Tab. 2 Comparison between simulation results and test data

\begin{tabular}{|c|c|c|c|}
\hline $\begin{array}{c}\text { Rotational } \\
\text { speed } \\
{[\mathbf{r p m}]}\end{array}$ & \multicolumn{3}{|c|}{$\begin{array}{c}\text { Pressure ratio @ surge condition } \\
\text { (peak of the curve) }\end{array}$} \\
\hline & Model & Test data & Error [\%] \\
\hline 7000 & 1.21 & 1.2 & $-0.8 \%$ \\
\hline 9000 & 1.33 & 1.34 & $-0.7 \%$ \\
\hline 10,000 & 1.42 & 1.42 & - \\
\hline 11,000 & 1.52 & 1.51 & $-0.7 \%$ \\
\hline 12,000 & 1.63 & 1.62 & $-0.6 \%$ \\
\hline 13,000 & 1.75 & 1.74 & $-0.6 \%$ \\
\hline
\end{tabular}

a

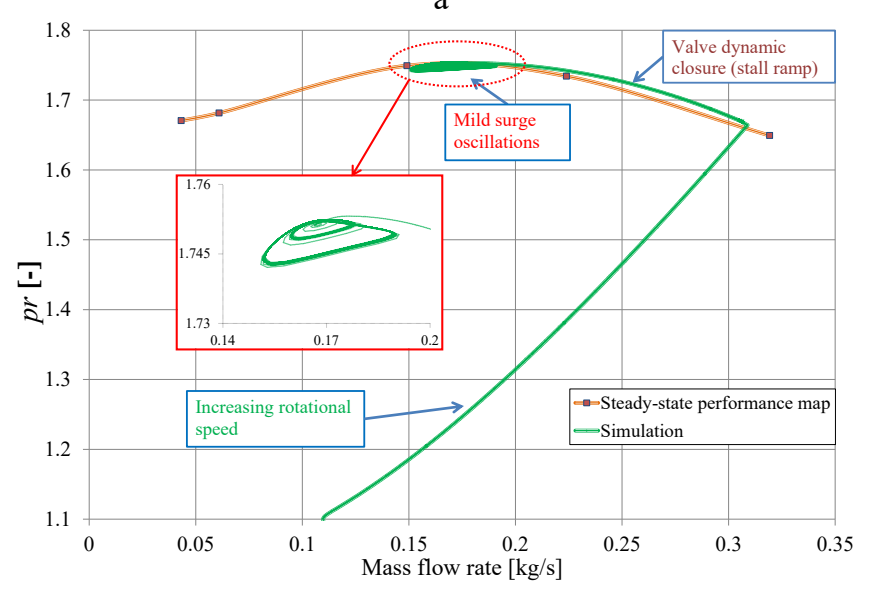

b

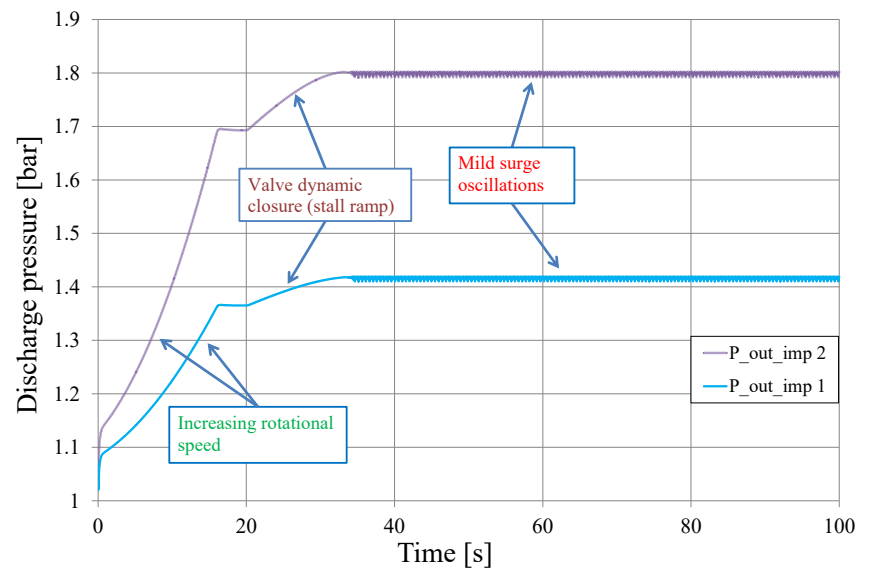

Fig. 7. Model results @13,000 rpm: a) operating point path (comparison with steady state experimental curve); b) discharge pressure of the impellers

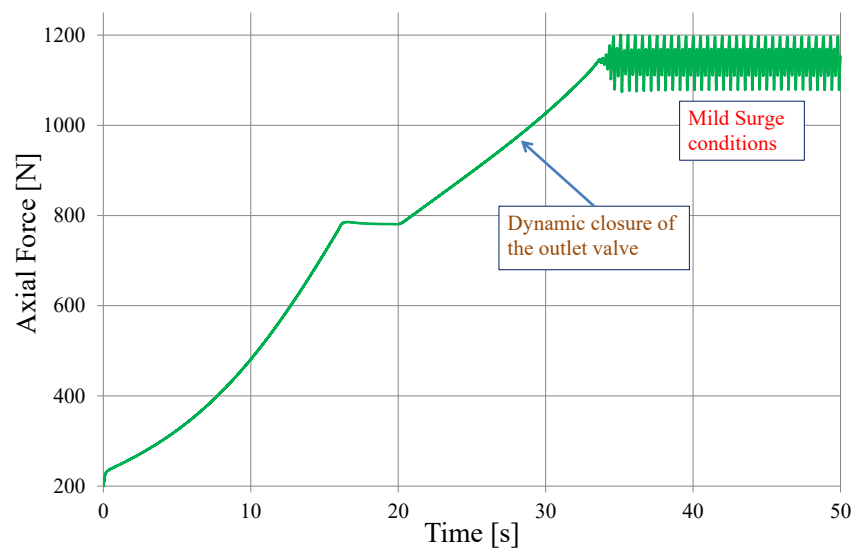

Fig. 8. Model results@13,000 rpm: net axial force, $F_{\mathrm{ax}, \mathrm{B}}$ acting on the bearing

As can be seen, this simulation lasts only $50 \mathrm{~s}$ since this time range is considered sufficient for the complete development of surge and its dynamic phenomena. The trend of Fig. 8 represents the elastic-viscous reaction force of the bearing, $F_{\text {ax,B }}$, at $13,000 \mathrm{rpm}$, in response to the fluid-dynamic thrust and to the rotor mass dynamics. The first ramp is the result of the increasing of compressor speed (from $5000 \mathrm{rpm}$ to $13,000 \mathrm{rpm})$. After about $15 \mathrm{~s}$ the desired rotational speed is achieved. Then the valve starts to close, generating the increase in axial force, as expected, and leading the compressor towards surge. In surge, the axial force achieves an approximate mean value of $1100 \mathrm{~N}$, showing the typical oscillations which reflect the pressure and mass flow pulsations. Table 3 reports the representative results of the simulator in terms of axial force peak values in mild surge conditions at 13,000 rpm.

Figure 9 presents an additional exhaustive comparison of the axial force at $13,000 \mathrm{rpm}$. It is clear how the model is capable of predicting not only the average magnitude of the axial forces with considerable accuracy, but also the axial force oscillations. Table 4 shows a comparison between experimental data and simulation results in terms of averaged and peak-topeak values, and frequency. The percentage error, regarding the average value of force, and peak-to-peak force is around $14 \%$ and $5 \%$, respectively. Regarding the frequency, the percentage error is higher, but the absolute values are very close and can be considered consistent with each other. Moreover, taking into account the complex phenomena under investigation - the difficulty of simulating the same degree of surge generated during tests is very high, especially for mild surge - the simulation results can be considered in good agreement with experimental results. Hence, this comparison demonstrates the reliability of the model for centrifugal compressor dynamic modeling working in any given piping system. Since an evaluation at only one rotational speed is not sufficient to decide on the reliability of the model, the values of the axial force at the other rotational speeds were also analyzed. The evidence of the reliability of the model can be found in Fig. 10. 
Tab. 3. Simulation results in surge condition @13,000 rpm

\begin{tabular}{|c|c|c|}
\hline & \multicolumn{2}{|c|}{$\begin{array}{c}\text { Axial force peaks } \\
{[\mathbf{N}]}\end{array}$} \\
\hline \multirow{2}{*}{ Mild Surge } & $\max$ & 1197 \\
\cline { 2 - 3 } & $\min$ & 1080 \\
\hline
\end{tabular}

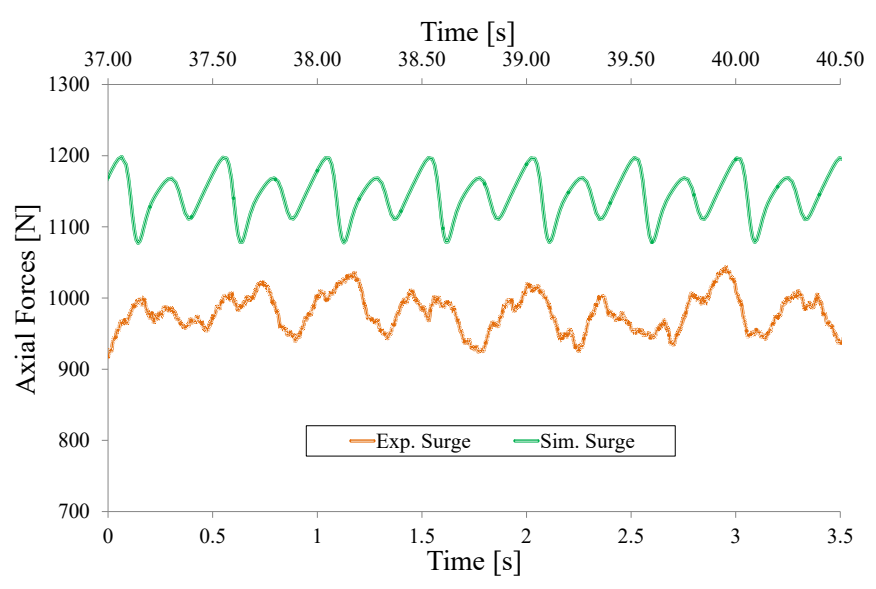

Fig. 9. Model validation: axial force oscillation during mild surge

Tab. 4. Comparison between simulation results and test data @ 13,000 rpm

\begin{tabular}{|c|c|c|c|c|}
\hline \multicolumn{2}{|c|}{} & $\begin{array}{c}\text { Average } \\
\text { axial force } \\
{[N]}\end{array}$ & $\begin{array}{c}\text { Peak-to- } \\
\text { peak axial } \\
\text { thrust [N] }\end{array}$ & $\begin{array}{c}\text { Frequency } \\
{[\mathrm{Hz}]}\end{array}$ \\
\hline \multirow{2}{*}{ Surge } & Simulator & 1120 & 117 & 4 \\
\cline { 2 - 5 } & Test Data & 980 & 111 & 2.5 \\
\hline \multicolumn{2}{|c|}{ Error [\%] } & $\sim 14 \%$ & $\sim 5 \%$ & $\sim 60 \%$ \\
\hline
\end{tabular}

Fig. 10a shows the results, in terms of peak-to-peak forces during surge, $F_{\text {peak-to-peak, }}$ obtained by the experimental tests and by the model, at different rotational speeds. It is clear that the magnitude of the results obtained by the model is highly consistent with that of the recorded data.

The model is less precise at a specific range of rotational speeds, 10,000-11,000 rpm, where the comparison shows a divergence of the results, although the absolute value of this difference is not significant (the dissimilarity is tiny, thus it is not dangerous when evaluating the load on a thrust bearing). This slight disagreement is due to a particular phenomenon, observed during the experimental tests. In fact, similar results were experimentally recorded at $9,000,10,000$ and $11,000 \mathrm{rpm}$, contrary to what could theoretically be expected (a rising axial force peak-to-peak was expected, by increasing the rotational speed). These experimental results can be explained by the nonhomogeneous degree of surge obtained at the different rotational speeds, due to the degree of closure of the valve. A proportional closure of the valve should be applied, at the different rotational speeds, to obtain a comparable degree of surge. Since during the experiments the valve closure was not always precisely and instantaneously stopped upon surge onset, it was difficult to exactly obtain the same values with the model. Similarly, Fig. 10b shows the surge frequency of the axial force recorded during tests and simulations, at the different rotational speeds. It is clear that the qualitative nature of the simulation results agrees with the experimental one. However, the absolute frequency values calculated by the model are always higher than those recorded in the test case. This can be justified by several factors: (i) the small volume of the test rig piping system, (ii) the model sketch chosen by the authors; (iii) the high unpredictability of mild surge; and (iv) the difficulty of the axial force frequency experimental evaluation. In fact, the model struggles to identify the correct frequency when the downstream volume is small, whereas it is quite precise if the size of the downstream piping system is larger (as demonstrated in [9]). Moreover, the sketch used for this piping system does not consider the effect of the outlet duct inertia at the compressor downstream but only the capacitance effect (the chosen model layout provides a plenum immediately after the compressor, and after the plenum a downstream valve).
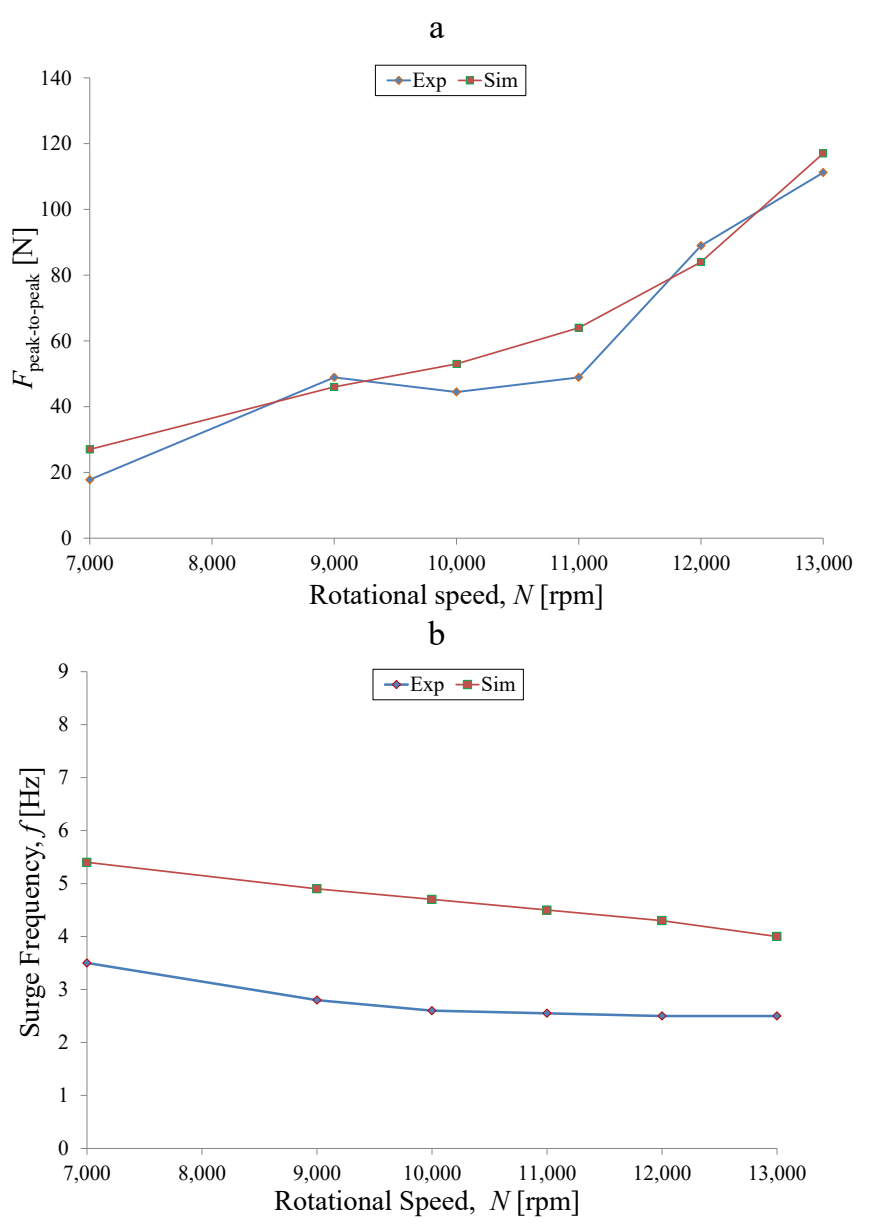

Fig. 10. Model validation: (a) peak-to-peak force; (b) frequency in mild surge condition 
This implies that the simulator tends to calculate a frequency higher than that recorded, especially with small downstream volumes.

Moreover, mild surge is the hardest degree of surge to simulate since it is the lowest level of axial-symmetric instability, and thus is the most affected by all the model inputs; the most important is the closure of the valve. In addition, the experimental measurement of axial force vibration frequency may be subject to a certain level of uncertainty due to electrical noise, the filtration of data, and the correct positioning of the load cells (incorrect positioning could result in misinterpretation of the carrier frequency).

\section{Deep surge}

This paragraph shows interesting model results regarding deep surge conditions. This instability is obtained by further reducing the mass flow rate in the model.

Figure 11a shows the dynamic compressor characteristic curve in terms of differential pressure $p_{\mathrm{amb}}-p_{\mathrm{P}}$ (since it provides the clearest representation of the deep surge cycle) during the deep surge process, whereas in Fig.11b the axial force oscillations are shown. It is evident that mild surge arises as a first instability and then a transition to deep surge occurs. These results cannot be compared to recorded data since such test might severely damage the compressor, however the thermodynamic results (Fig. 11a) are quite reliable since a validation of the preliminary and simplified version of this model was carried out in [9].

The force results generated in deep surge cannot be validated, but they seem highly consistet with what is expected to occur - in deep surge the amplitudes of the forces increase while the frequency decreases - see Fig. 11b (future work will focus on this theme).

\section{Sensitivity analysis}

In order to understand more clearly which parameters affect surge severity the most, in terms of axial forces acting on the thrust bearing, a sensitivity analysis was carried out.

The analysis focuses on the bearing stiffness $k$, the rotor mass $M_{\text {rot }}$, and the compressor downstream volume $V_{\mathrm{p}}$. The aim is to evaluate the potential effect of these quantities on $F_{\text {peak,max }}$, $F_{\text {peak,min }}$ and $F_{\text {peak-to-peak, }}$, respectively the maximum, minimum and peak-to-peak values of the force. Obviously, also the rotational speed $N$ of the compressor (not presented in this analysis) during operation plays a very important role due to the pressure ratio and impeller work that characterize the compressor when the peak of the curve is achieved, i.e. before surge. Another very significant parameter, which affects the calculation of the forces, is the radius of the impellers $r_{\text {disch. }}$. Regarding this parameter, it is difficult to analyze its effect only by varying it in the model as an independent parameter, since it would affect the compressor performance curve, thus the values of pressure ratio and work.

In Fig. 12 the peak-to-peak values of the net axial force are plotted, alongside the maximum and minimum peaks, against the volume between the compressor and the control valve. a

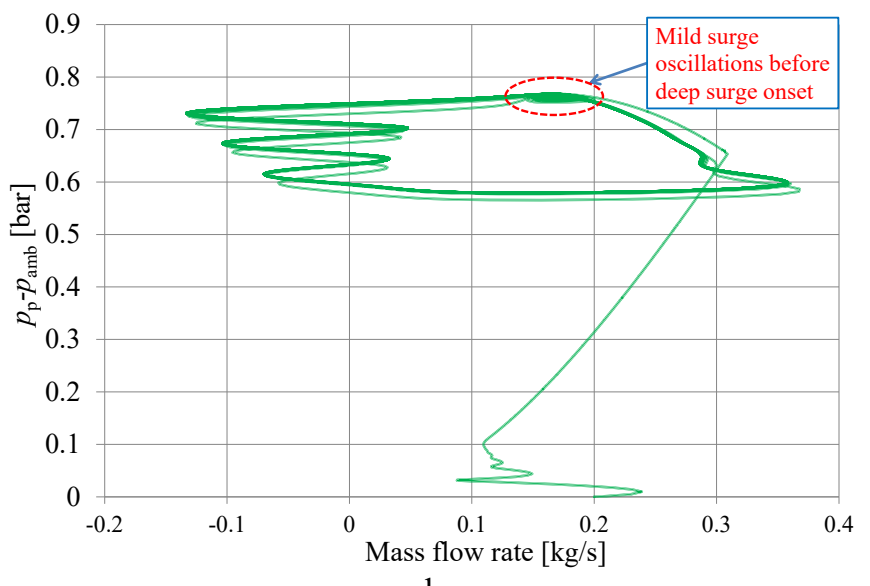

b

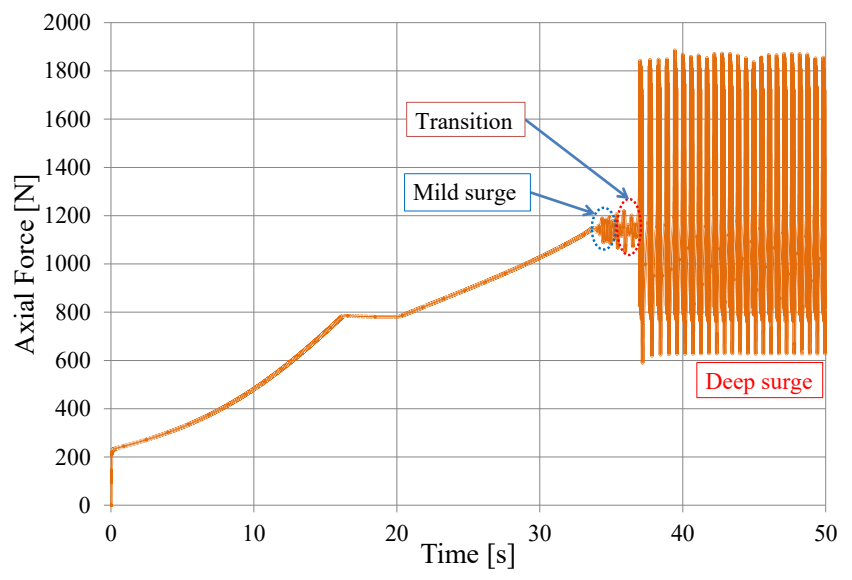

Fig. 11. Deep surge simulation@13,000 rpm: compressor characteristic curve and net axial force $F_{\mathrm{ax}, \mathrm{B}}$

The figure shows the increase in the maximum peak and peak-to-peak values with the volume, and the decrease in the minimum peak values. This trend continues until a certain volume, which is the dimension that generates the maximum surge cycle, after which the force values remain similar.

Figure 13 shows the influence of the stiffness, $k$, of the bearing by keeping the clearance constant. The results refer to a $1 \mathrm{~m}^{3}$ downstream volume so that deep surge is achieved. It can be stated that the higher the stiffness, the less dangerous the surge, for the thrust bearing. The decrease in $k$ results in an approximately constant trend of $F_{\text {peak,max }}$ and $F_{\text {peak-to-peak }}$ until about $30 \cdot 10^{6} \mathrm{~N} / \mathrm{m}$. However, by further diminishing $k$, these values decrease; which would mean that in that region, i.e. for lower values of $k$, the loads are minimized. This fact can be misleading because, by reducing $k$, the maximum elastic force, $F_{\text {el,max }}$, of the bearing also goes down, and this increases the danger of plastic deformation, and subsequent destruction of the bearing. On the other hand, a bearing with a high level of stiffness could generate and transmit vibrations to the mechanical and aerodynamic components; which is not desirable in any application. Figure 14 illustrates the effect of 
the rotor mass on the axial force oscillations acting on the bearing. Also in this case the results refer to a $1 \mathrm{~m}^{3}$ downstream volume.

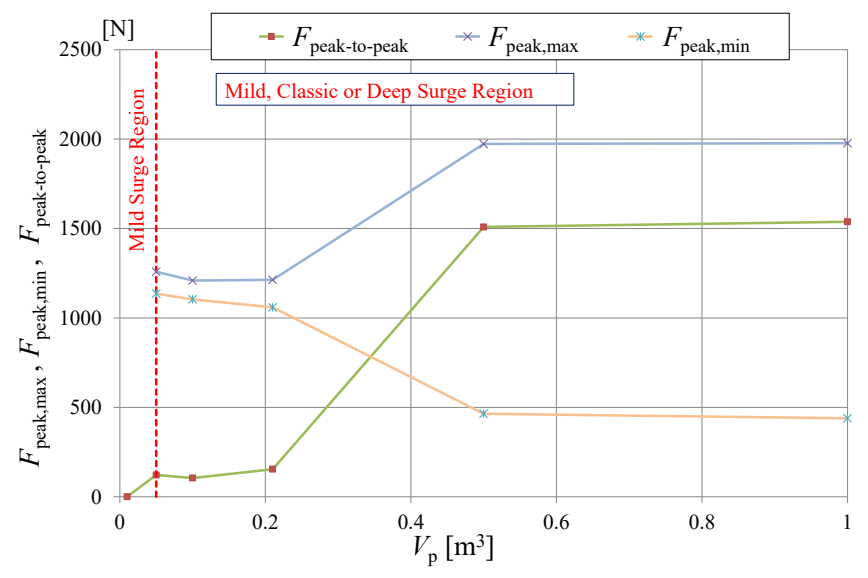

Fig. 12. Influence of the compressor downstream volume on the axial force

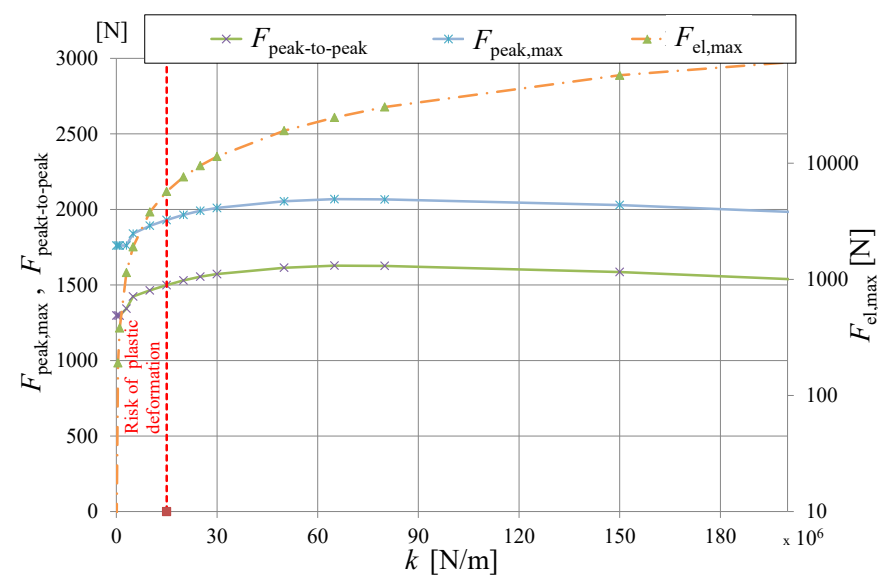

Fig. 13. Influence of the bearing stiffness on the axial force

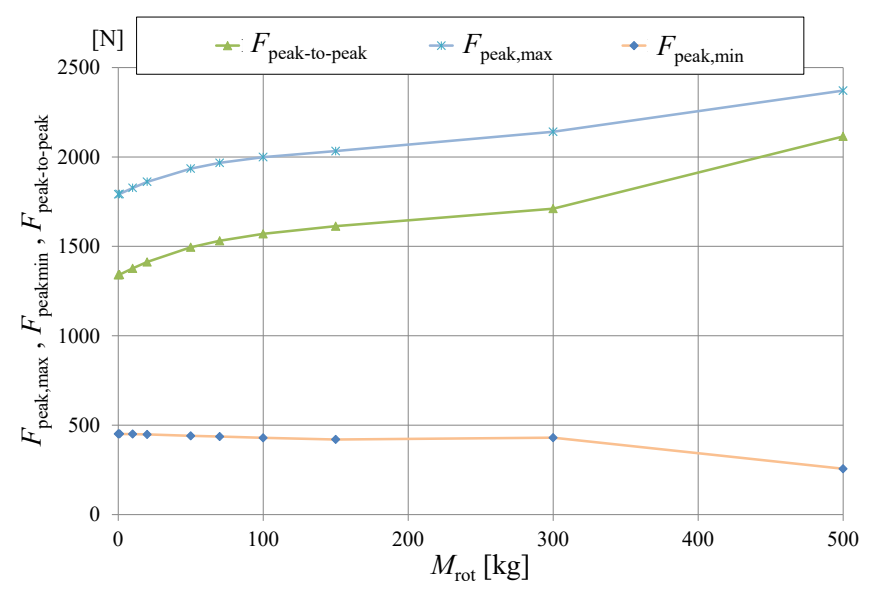

Fig. 14. Influence of the rotor mass on the axial forces
The increase in rotor mass leads to an increase in force amplitudes. This phenomenon was not observed when analyzing smaller oscillations (mild surge) since those fluctuations are more rapid, in terms of frequency, and less strong in terms of amplitude. Therefore, in mild surge conditions, the mass of the rotor did not exhibit any substantial influence on the loads acting on the bearing. In fact, the higher the amplitude of the exciting fluid-dynamic force, the more significant the acceleration of the mass. Considering that (i) a general force is given by mass multiplied by acceleration, (ii) usually, a higher mass is subject to lower acceleration (especially in this particular case), and (iii) the influence of the acceleration on the resultant force is generally lower than that of the mass; it is feasible to state that the higher the mass, the more this mass affects the force produced by the rotor movement (in the axial direction). This fact implies that if the rotor is moved by the fluid-dynamic thrust generated on the impellers, the higher the rotor mass, the higher the maximum peak and the peak-to-peak values.

On the other hand, it could signify a high moment of inertia and thus an advantage in case of emergency shutdown, since the rotor would slow down very gently, limiting the inception of surge.

\section{CONCLUSIONS}

In this paper a model for the prediction of the dynamic behavior of a compressor, in particular during surge, is proposed. This model is based on the bond graph approach which allows a modular structure and can be applied to many engineering systems.

The study of the axial force focuses on the main contributions given in a single impeller so that the model can also be applied to multistage compressors. The simulator can manage several types of gases and mixtures.

The model was applied to the SwRI test case by implementing the compressor curves and the geometric dimensions alongside the layout of the piping system. A comparison between the experimental data and the simulator response, at a rotational speed of 13,000 rpm, was carried out, demonstrating the accuracy of the model (and its modular structure), which has proved to be strongly successful in simulating surge. In fact, the dynamic characteristic curve obtained by the model successfully resembles the real steadystate curve of the compressor and the surge, in this case mild surge, appears immediately after the characteristic curve peak. Moreover, the simulated trend of the axial force on the thrust bearing was shown to be highly consistent with the experimental data acquired in surge conditions. Additional comparisons with experimental data were carried out at the other rotational speeds tested. The validation showed that the model succeeds in determining values of surge frequency (the model showed qualitative agreement but the values were slightly higher) and axial thrust values, very close to the ones recorded during testing.

A deep surge condition scenario predicted by the model is also reported. The simulation showed the high increase of the 
surge cycle, which generates a significant increase in axial thrust oscillations. This analysis of the deep surge condition could not be validated since running the compressor in deep surge would likely damage the compressor itself and its components. However, a preliminary version of this model (developed in a previous work) was shown to be highly reliable in simulating the thermodynamic of deep surge.

A sensitivity analysis demonstrated that the increase in downstream volume led to an increase in axial forces on the bearing during surge. This effect is valid up to a definite volume value, which can be identified as the volume that causes the maximum surge cycle.

Moreover, the value of the bearing stiffness was confirmed to be a fundamental factor to be taken into account. The surge severity was shown to decrease with the increase in stiffness despite the consequent rise in vibrations.

As a general rule it can also be stated that, the higher the rotor mass, the higher the axial force peaks are. This effect was clear in deep surge conditions, due to higher pressure fluctuations and their frequency, which allowed greater movement in the axial direction of the mass, thus generating a higher bearing reaction force.

Further discussion is required on the subject of fatigue, which was not covered in this work.

This model can therefore be considered a powerful instrument for simulating the thermodynamic behavior of the compressor and predicting the axial thrust which acts on the thrust bearing in surge, but also in generic transients. This is a precious contribution to industry since it increases the understanding of the fatigue forces acting on the bearing, in order to predict and prevent maintenance, or repairs, but also for providing additional guidelines for the correct selection, or design, of the thrust bearing.

Despite the great strength of the model presented in this paper, some improvements are still needed to overcome its current limits. The next step of this research will be the accurate calculation of the core rotation factor along the gaps, in order to make the model also suitable for high pressure compressors.

\section{REFERENCES}

[1] Rasmussen, Peter C., Kurz, R. Centrifugal Compressor Applications - Upstream and Midstream. (2009) 38th Turbo Machinery Symposium, Houston, TX, pp. 14-17.

[2] Kurz, R., and Brun, K. Site Performance Test Evaluation for Gas Turbine and Electric Motor Driven Compressors. (2005) Proceedings of the Thirty-Fourth Turbomachinery Symposium, pp. 53-62.

[3] Kurz, R.. Gas Turbine Performance. (2005) Proceedings of the Thirty-Fourth Turbomachinery Symposium, Houston, TX.

[4] Boyce, Meherwan P. (2003) Centrifugal Compressors: A Basic Guide. PlennWell Books.

[5] Moore, J.J., Ransom, D.L., Viana, F. Rotordynamic Force Prediction of Centrifugal Compressor Impellers Using Computational Fluid Dynamics. (2011) Journal of
Engineering for Gas Turbines and Power, 133 (4), art. no. 042504. DOI:10.1115/1.2900958.

[6] Lüddecke, B., Nitschke, P., Dietrich, M., Filsinger, D., Bargende, M. Unsteady Thrust Force Loading of a Turbocharger Rotor During Engine Operation. (2016) Journal of Engineering for Gas Turbines and Power, 138 (1), art. no. 012301. DOI: 10.1115/1.4031142.

[7] Kurz, R., Marechale, R.K., Fowler, E.J., Min Ji,. Cave, M.J. Operation of Centrifugal Compressors in Choke Conditions. (2011) Proceedings of the Fortieth Turbomachinery Symposium, Houston, Texas.

[8] Cumpsty, N.A. (1989) Compressor Aerodynamics. Longman Scientific \& Technical.

[9] Munari, E., Morini, M., Pinelli, M., Spina, P.R. Experimental Investigation and Modeling of Surge in a Multistage Compressor. (2016) $8^{\text {th }}$ International Conference on Applied Energy (ICAE).

[10] Brun, K., Simons, S., Kurz, R., Pinelli, M., Munari, E., Morini, M. Measurement and Prediction of Centrifugal Compressor Axial Forces During Surge - part 1: Test and Force Measurement Results. Submitted to ASME Turbo Expo 2017, GT2017-63061.

[11] Greitzer, E.M. Surge and Rotating Stall in Axial Flow Compressors - Part I: Theoretical Compression System Model. (1976) Journal of Engineering for Power, 98(2), pp. 190-198.

[12] Morini, M., Pinelli, M., Venturini, M. Development of a One-Dimensional Modular Dynamic Model for the Simulation of Surge in Compression Systems. (2007) Journal of Turbomachinery, 129(3), pp. 437-447. DOI: 10.1115/1.2447757.

[13] Galindo, J., Serrano, J.R., Climent, H., Tiseira, A. Experiments and Modelling of Surge in Small Centrifugal Compressor for Automotive Engines. (2008) Experimental Thermal and Fluid Science, 32(3), pp. 818-826. DOI: 10.1016/j.expthermflusci.2007.10.001

[14] Vepa, R. Modelling and Quasilinear Control of Compressor Surge and Rotating Stall Vibrations (2010) Mathematical Problems in Engineering, art. no. 314172. DOI: $10.1155 / 2010 / 314172$

[15] Moore, F.K., Greitzer, E.M. A Theory of Post-Stall Transients in Axial Compression Systems: Part I Development of Equations. (1986) Journal of Engineering for Gas Turbines and Power, 108(1), 68-76.

[16] Day, I.J. Stall, Surge, and 75 Years of Research. (2016) Journal of Turbomachinery, 138(1), art. no. 011001. DOI: $10.1115 / 1.4031473$.

[17] Pearson, H., Mc Kenzie, A.B. Wakes in Axial Compressors. (1959) Journal of the Royal Aeronautical Society, 63, pp.415-416.

[18] Cousins, W.T., Davis Jr., M.W. Evaluating Complex Inlet Distortion with a Parallel Compressor Model: Part 1 - Concepts, Theory, Extensions, and Limitations. (2011) Proceedings of the ASME Turbo Expo, 1, pp. 112. DOI: $10.1115 / \mathrm{GT} 2011-45067$.

[19] Mazzawy, R.S. Multiple Segment Parallel Compressor 
Model for Circumferential Flow Distortion. (1977) $J$ Eng Power, Trans ASME, 99 Ser A (2), pp. 288-296.

[20] Floyd, R.S., Jr., Davis, M., Jr. Validation of a Modified Parallel Compressor Model for Prediction of the Effects of Inlet Swirl on Compressor Performance and Operability. (2015) Proceedings of the ASME Turbo Expo, 1. DOI: 10.1115/GT2015-43415.

[21] Grong, T. S. Modeling of Compressor Characterisics and Active Surge Control. (2009) Student Thesis, Norwegian University of Science and Technology.

[22] Belardini, E., Pandit, R., Satish Koyyalamudi, V.V.N.K., Rubino, D.T., Tapinassi, L. 2nd Quadrant Centrifugal Compressor Performance: Part II. (2016) Proceedings of the ASME Turbo Expo, 9. DOI: 10.1115/GT2016-57124.

[23] Leufvén, O., Eriksson, L. A Surge and Choke Capable Compressor Flow Model-Validation and Extrapolation Capability. (2013) Control Engineering Practice, 21(12), pp. 1871-1883.

[24] Kilchyk, V., Abdelwahab, A., Rosinski, A. Application of the Surge Model to Radial Compressor System Cycle Optimization. (2013) Proceedings of the ASME Turbo Expo, 6 C, art. no. GT2013-94222.

[25] Yoon, S.Y., Lin, Z., Lim, K.T., Goyne, C., Allaire, P.E. Model Validation for an Active Magnetic Bearing Based Compressor Surge Control Test Rig. (2010) Journal of Vibration and Acoustics, Transactions of the ASME, 132(6), art. no. 061005. DOI: 10.1115/1.4001845.

[26] Domm, U., Zilling, H. Axial Thrust in Centrifugal Pumps. (1966) IAHR Symposium Pumps in Power Stations, pp. J23-J34, Braunschweig.

[27] Kurokawa, J., Toyokura, T. Study on the Axial Thrust of the Radial Flow Turbomachinery. (1972) The second International JSME Symposium, Fluid Machinery and Fluidics, Tokyo.

[28] Mazzawy, R.S. Surge Induced Structural Loads in Gas Turbines. (1980) Journal of Engineering for Power, 102(1), pp. 162-168.

[29] Bondarenko, G.A., Ganelin, B.Ya., Marufenko, T.M. Method of Calculating Axial Forces in a Centrifugal Compressor Based on a Refined Model of Flow. (1992) Chemical and Petroleum Engineering, 28(2), pp. 81-85. DOI: 10.1007/BF01148826.

[30] Baldassarre, L., Bernocchi, A., Rizzo, E., Fontana, M., Maiuolo, F. Axial Thrust in High Pressure Centrifugal Compressors: Description of a Calculation Model Validated by Experimental Data from Full Load Test. (2015) 44th Turbomachinery \& 31st Pump User Symposia, Huston, TX.

[31] Da Soghe, R., Facchini, B., Innocenti, L., Micio, M. Analysis of Gas Turbine Rotating Cavities by an OneDimensional Model. (2009) Proceeding of ASME Turbo Expo, Power for Land, Sea, and Air, pp. 355-364. DOI: 10.1115/GT2009-59185.

[32] Han, Z.-X., Cizmas, P.G.A. A CFD Method for Axial Thrust Load Prediction of Centrifugal Compressors. (2003) International Journal of Turbo and Jet Engines,
20(1), pp. 1-16.

[33] Bidaut, Y., Dessibourg, D. The Challenge for the Accurate Determination of the Axial Rotor Thrust in Centrifugal Compressors. (2014) 43rd Turbomachinery \& 30th Pump Users Symposia (Pump \& Turbo 2014), Houston, TX.

[34] Baumann, U. Rotordynamic Stability Tests on HighPressure Radial Compressors. (1999) Proceedings of the 28th Turbomachinery Symposium, Turbomachinery Laboratory, Texas A\&M University, College Station, TX.

[35] Bidaut, Y., Baumann, U., \& Al-Harthy, S. M. H. Rotordynamic Stability of a 9500 psi Reinjection Centrifugal Compressor Equipped with a Hole Pattern Seal-Measurement versus Prediction Taking into Account the Operational Boundary Conditions. (2009) Proceeding. of 38th Turbomachinery Symposium, Texas, pp. 251-259.

[36] Petry, N., König, S., Benra, F.-K. Influence of the Swirling Flow in the Side Cavities of a High-Pressure Centrifugal Compressor on the Characteristics of Excited Acoustic Modes. (2013) Journal of Turbomachinery, 135(3), art. no. 031024. DOI: 10.1115/1.4007544.

[37] Roy, R. P., Devasenathipathy, S., Xu, G., \& Zhao, Y. A Study of the Flow Field in a Model Rotor-Stator Disk Cavity. (1999) ASME International Gas Turbine and Aeroengine Congress and Exhibition, pp. V003T01A064-V003T01A064.

[38] Li, W. Model of Flow in the Side Chambers of an Industrial Centrifugal Pump for Delivering Viscous Oil. (2013) Journal of Fluids Engineering, Transactions of the ASME, 135(5), art. no. 051201. DOI: 10.1115/1.4023664.

[39] Will, B. C. Theoretical, Numerical and Experimental Investigation of the Flow in Rotor-Stator Cavities With Application to a Centrifugal Pump. (2011) Doctoral Dissertation, Universität Duisburg-Essen, Fakultät für Ingenieurwissenschaften» Maschinenbau und Verfahrenstechnik» Institut für Energie-und Umweltverfahrenstechnik.

[40] Childs, P.R. Rotating Flow. (2011) ButterworthHeinemann, Oxford.

[41] Hu, J.-P., Li, K.-J. Thermal-Hydraulic Modeling and Analysis of Hydraulic System by Pseudo-Bond Graph. (2015) Journal of Central South University, 22(7), pp. 2578-2585. DOI: 10.1007/s11771-015-2787-0.

[42] Brown, F. T. (2006) Engineering System Dynamics: a Unified Graph-Centered Approach. CRC press.

[43] Uddin, N., Gravdahl, J.T. Bond Graph Modeling of Centrifugal Compression Systems. (2015) Simulation, 91(11), pp. 998-1013. DOI: 10.1177/0037549715612124.

[44] Krikelis, N.J., Papadakis, F. Gas Turbine Modelling Using Pseudo-Bond Graphs. (1988) International Journal of Systems Science, 19(4), pp. 537-550. DOI: 
$10.1080 / 00207728808967624$.

[45] Movaghar, A. S., Novinzadeh, A. Ideal Turbo Charger Modeling and Simulation Using Bond Graph Approach. (2011) Proceeding of ASME Turbo Expo, pp. 871-879.

[46] Munari, E., Morini, M., Pinelli, M., Spina, P.R., Suman, A. Experimental Investigation of Stall and Surge in a Multistage Compressor. (2017) Journal of Engineering for Gas Turbines and Power, 139(2), art. no. 022605. DOI: $10.1115 / 1.4034239$.

[47] Rohsenow, W.M., Hartnett, J. P., Cho, Y. I. (1998). Handbook of Heat Transfer. (Vol. 3). New York: McGraw-Hill.

[48] Dixon, S.L., \& Hall, C. Fluid Mechanics and Thermodynamics of Turbomachinery. (2013). Butterworth-Heinemann.

[49] Berdanier, R.A., Smith, N.R., Fabian, J.C., Key, N.L. Humidity Effects on Experimental Compressor Performance-Corrected Conditions for Real Gases. (2015) Journal of Turbomachinery, 137(3), art. no. 031011. DOI: 10.1115/1.4028356.

[50] Belardini, E., Rubino, D.T., Tapinassi, L., Pelella, M. Modeling of Pressure Dynamics During Surge and ESD. (2015) $3^{\text {rd }}$ Middle East Turbomachinery Symposium, Doha, Quatar.

[51] Gravdahl, J.T., Egeland, O. Compressor Surge and Rotating Stall: Modeling and Control. (2012) Springer Science \& Business Media.

[52] Jiang, W., Khan, J., Dougal, R.A. Dynamic Centrifugal Compressor Model for System Simulation. (2006) Journal of Power Sources, 158(2), 1333-1343.

[53] Ferrara, E.G. Characterization of Vaneless Diffuser Rotating Stall on Centrifugal Compressors for Industrial Applications. (2013) PhD Thesis, University of Florence, Florence.

[54] Japikse, D. Centrifugal Compressor Design and Performance. (1996) Wilder, VT: Concepts ETI, Inc.

[55] Lüdtke, K.H. Process Centrifugal Compressors: Basics, Function, Operation, Design, Application. (2013) Springer Science \& Business Media.

[56] Gülich, J.F. Centrifugal Pumps. (2008) Berlin, Springer.

[57] Tibboel, G.A. Modification of a One-Dimensional Dynamic Compression System Model to Calculate Stage Characteristics Internally. (2003) Student Thesis, University of Tennessee, Knoxville.

[58] Casey, M., Robinson, C.A Method to Estimate the Performance Map of a Centrifugal Compressor Stage. (2013) Journal of turbomachinery, 135(2), 021034. 